\title{
Design, Synthesis and Antibacterial Activity Studies of Novel Quinoline Carboxamide Derivatives
}

\author{
Yellappa Shivaraj, Malenahalli H. Naveen, Giriyapura R. Vijayakumar ${ }^{\dagger}, *$, and Doyijode B. Aruna Kumar ${ }^{\dagger}$ \\ Department of Chemistry, Government Science College, Bangalore-560001, India \\ 'Department of Chemistry, Tumkur University, Tumkur-572103, India. \\ *E-mail: vijaykumargr18@yahoo.co.in
}

(Received November 27, 2012; Accepted March 13, 2013)

\begin{abstract}
A series of novel quinoline-6-carboxamides and 2-chloroquinoline-4-carboxamides were synthesized by the reaction of their analogous carboxylic acids with various amine derivatives in the presence of base TEA and protecting agent BOP at room temperature. Synthesized compounds were confirmed by spectral characterization viz IR, ${ }^{1} \mathrm{H}-\mathrm{NMR}$, and MS. Antibacterial activity carried out against Escherichia coli and Staphyllococcus aureus indicated that the synthesized compounds were active against these microorganisms.
\end{abstract}

Key words: Quinoline-6-carboxamides, 2-Chloro quinoline-4-carboxamides, Antibacterial activity

\section{INTRODUCTION}

A quinoline ring containing compounds exhibit potent biological activities and has been proved by number of recent reports. Quinoline derivatives were synthesized and explored for their analgesic activity, ${ }^{1}$ as antiallergetic agents, ${ }^{2}$ in treating alzheimer's disease (AD), ${ }^{3}$ as anticancer, ${ }^{4,5}$ antitinephritic, ${ }^{6}$ antitumor ${ }^{7}$ and anti-inflammatory activities. A series of 2-styrylquinolines and quinoline-2carboxamides were identified as potent inhibitors of the Pim-1 kinase which is involved in the control of cell growth, differentiation, proliferation and apoptosis. ${ }^{4}$ The $\mathrm{Cu}(\mathrm{II})-$ quinoline carboxamide complexes were found to exhibit cytotoxicity against murine leukemia P-388 and human leukemia HL-60 cell lines, which is more potent than cisplatin. ${ }^{5}$ Linomide-related quinoline-3-carboxamides and their analogues were found to be exhibit effective antinephritic activities. ${ }^{6}$ In vivo and in vitro studies of 7-Oxo7H-naphtho[1,2,3-de]quinoline-11-carboxamides and analogues proved that these compounds have greater antitumor activity. ${ }^{7}$ A series of quinoline carboxamide derivatives have also been evaluated for positive inotropic activity, ${ }^{8}$ as potential radio ligands in the neurodegeneration systems ${ }^{9}$ and antiviral against acyclovir resistant herpes simplex virus. $^{10}$

We inspired by the above potent pharmacological properties of these quinoline ring containing compounds and have been synthesized some novel quinoline carboxamides derivatives and explored their biological activity.

\section{RESULTS AND DISCUSSION}

Here in we report a synthesis and characterization of series of 2-chloro quinoline-4-carboxamides and quinoline6-carboxamides. Both these analogous of compounds were synthesized by the reaction of respective carboxylic acid with various amine derivatives in the presence of base TEA and protecting agent BOP at room temperature. Even though there are reports for the synthesis of quinoline carboxamides, the novelty of this paper is employing of new variety of amine derivatives and one such compound is the pyrimidine cyclopropyl amine hydrochloride which has been synthesized from cyclopropanone as described in Scheme 1. We found that DMF has good solvent and could be employed at room temperature to afford good yield of the quinoline carboxamides. A report of various amine derivatives employed, product quinoline carboxamide structure and reaction yield has been given in Table 1. All the synthesized final compounds were purified by recrystalization and confirmed by spectral characterization using IR, ${ }^{1} \mathrm{H}-\mathrm{NMR}$, and LCMS.

Keto quinoline carboxylic acid, $\mathbf{1}$ was converted to chloro quinoline carboxylic acid, $\mathbf{2}$ by the reaction of $\mathbf{1}$ with $\mathrm{POCl}_{3}$ in DMF solvent. The reaction mixture stirred at $100{ }^{\circ} \mathrm{C}$ for 12 hours could afford product 2 in $90 \%$ yield. Coupling of the carboxylic acid $\mathbf{2}$ with amine derivative using coupling reagent $\mathrm{BOP}$ in the presence of base triethyl amine and in the solvent DMF at room temperature gave the product $\mathbf{2}$ a-f $($ Scheme 2$)$. The carboxylic acid $\mathbf{3}$ was converted in to carboxamide derivatives $\mathbf{3}$ a-d by 


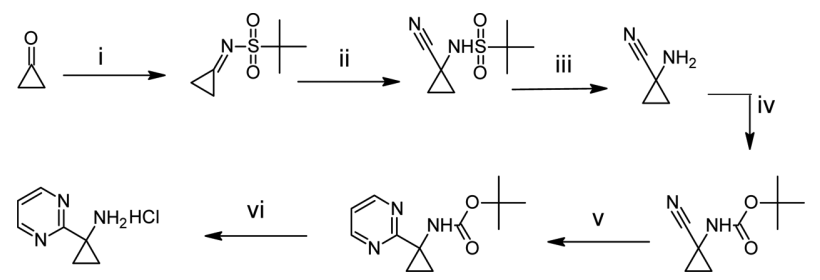

Scheme 1. Preparation of 1-(pyrimidin-2-yl)cyclopropanamine Hydrochloride. Reagents and conditions: i) 2-methyl propane-2sulphanamide, Titanium (IV) isopropaxide, THF, $60^{\circ} \mathrm{C}, 12 \mathrm{~h}$. ii) TMS-CN, Titanium (IV) isopropaxide, DCM, RT, 12h iii) Dioxane-HCl, Dioxane, $0^{\circ} \mathrm{C}$. iv) TEA, di-tertiary butyl dicarbamate, Dioxane, $0{ }^{\circ} \mathrm{C}, 12 \mathrm{~h}$ v) $\mathrm{N}$-acetyl L-cystene, Ammonium acetate, Methanol, $75^{\circ} \mathrm{C}, 14 \mathrm{~h}$; NN-dimethyl amino2-propen-3-al, Sodium methoxide, $75^{\circ} \mathrm{C}, 24 \mathrm{~h}$. vi) Dioxane- $\mathrm{HCl}$, Dioxane, $0^{\circ} \mathrm{C}, 2 \mathrm{~h}$.

Table 1. Synthesis of quinoline carboxamide derivatives ${ }^{a}$

Entry Carboxylic acid

${ }^{a}$ Carboxylic acid (1 mmol), R-NH 2 (1.5 mmol), BOP (1.5 mmol), TEA (5 mmol), RT, $12 \mathrm{~h}$.

${ }^{b}$ Isolated yield.

using similar reaction conditions which could employed for 2 (Scheme 3).

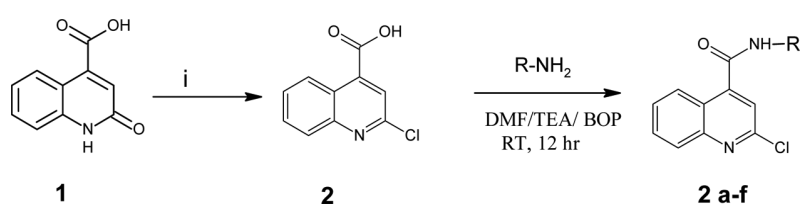

Scheme 2. Synthesis of 2-chloro quinoline-4-carboxamides i) $\mathrm{POCl}_{3}, \mathrm{DMF}, 100{ }^{\circ} \mathrm{C}, 4 \mathrm{~h}$.

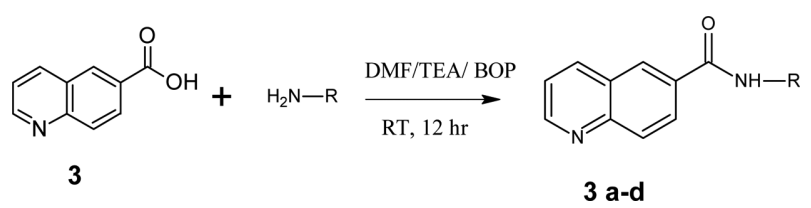

Scheme 3. Synthesis of Quinoline-6-carboxamides.

Table 2. Screening of quinoline carboxamide derivatives for antibacterial activity ${ }^{a}$

\begin{tabular}{cccc}
\hline \multirow{2}{*}{ Entry } & \multirow{2}{*}{ Compound } & \multicolumn{3}{c}{ Bacterial Strains } \\
\cline { 3 - 4 } & & Escherichia coli & Staphylococcus aureus \\
\hline 1 & $\mathbf{2 a}$ & Ac & Ac \\
2 & $\mathbf{2 b}$ & Ac & Ac \\
3 & $\mathbf{2 c}$ & Ac & Ac \\
4 & $\mathbf{2 d}$ & Ia & Ia \\
5 & $\mathbf{2 e}$ & Ac & Ac \\
6 & $\mathbf{2 f}$ & Ia & Ac \\
7 & 3a & Ac & Ac \\
8 & 3b & Ac & Ac \\
9 & 3c & Ac & Ac \\
10 & 3d & Ia & Ia \\
\hline${ }^{a}$ Compounds concentration $-2 \mathrm{mg} / \mathrm{ml} ;$ Ac-active; Ia-inactive
\end{tabular}

\section{Screening for Antibacterial Activity}

The synthesized carboxamides were tested for antibacterial activity against two bacteria viz., Escherichia coli and Staphylococcus aureus. The preliminary study was carried out using spread plate method. ${ }^{11,12}$ Compounds tabulated in Table 2 were shown activity against the tested microorganisms. Compounds which have shown antibacterial activity were subjected for Minimum Inhibitory Concentration (MIC) studies and results were given in Table 3.

\section{CONCLUSION}

In the present work we synthesized series of 2-chloroquinoline-4-carboxamides (2a-f) and quinoline-6-carboxamides (3a-d) and evaluated in vitro antibacterial activities against Escherichia coli and Staphyllococcus aurens. Results indicated that all the synthesized compounds (except 2d and 3d) show activity against tested microorganisms in milligram level. 
Table 3. Determination of Minimum Inhibitory Concentration (MIC) values for the quinoline carboxamide derivatives

\begin{tabular}{|c|c|c|c|c|c|c|c|c|c|}
\hline \multirow{2}{*}{$\begin{array}{c}\text { Bacterial } \\
\text { Strains }\end{array}$} & \multirow{2}{*}{$\begin{array}{l}\text { Concentration } \\
(\mathrm{mg} / \mathrm{ml})\end{array}$} & \multicolumn{8}{|c|}{ Tested compounds } \\
\hline & & $2 \mathrm{a}$ & $2 b$ & $2 \mathrm{c}$ & $2 \mathrm{e}$ & $2 f$ & $3 a$ & $3 b$ & $3 c$ \\
\hline \multirow{5}{*}{ E. coli } & 0.5 & + & - & + & + & + & + & + & + \\
\hline & 1.0 & + & - & + & + & + & + & + & - \\
\hline & 1.5 & + & - & - & + & + & + & - & - \\
\hline & 2.0 & - & - & - & - & + & - & - & - \\
\hline & 2.5 & - & - & - & - & + & - & - & - \\
\hline \multirow{5}{*}{ S. aureus } & 0.5 & + & + & + & + & + & + & + & + \\
\hline & 1.0 & + & + & + & + & + & + & + & + \\
\hline & 1.5 & - & + & + & + & + & - & - & + \\
\hline & 2.0 & - & - & - & - & - & - & - & - \\
\hline & 2.5 & - & - & - & - & - & - & - & - \\
\hline
\end{tabular}

+: Presence of growth; -: Absence of growth

\section{EXPERIMENTAL}

The uncorrected melting points of compounds were taken in an open capillary using an electrical heating melting point apparatus. IR spectra were recorded on PerkinElmer FT spectrophotometer in $\mathrm{KBr}$ disc. ${ }^{1} \mathrm{H}-\mathrm{NMR}$ spectra were recorded using $400 \mathrm{MHz}$ Bruker NMR spectrometer in DMSO- $d_{6}$ solvent and chemical shift values are expressed in ppm relative to TMS as an internal standard. LCMS spectra were recorded on LCMS-8030, Shimadzu.

\section{Procedure for the Preparation of 2-Chloroquinoline-} 4-carboxylic acid, 2

To a mixture of 2-oxo-1,2-dihydroquinoline-4-carboxylic acid, 1 (1 mmol), in DMF was added to the mixture of DMF $(2 \mathrm{mmol})$ and $\mathrm{POCl}_{3}(2 \mathrm{mmol})$ at $0{ }^{\circ} \mathrm{C}$. The reaction mixture was stirred at $100{ }^{\circ} \mathrm{C}$ for $4 \mathrm{~h}$. Completion of the reaction was monitored by LC/MS. The reaction mass was diluted with ice cold water, a pale yellow solid precipitates out was filtered and dried at suction pump.

\section{General Procedure for the Preparation of 2-Chloro} quinoline-4-carboxamides, 2a-f

To a mixture of 2-chloroquinoline-4-carboxylic acid $\mathbf{2}$, $(1 \mathrm{mmol})$ in DMF was added amine $(1.5 \mathrm{mmol})$, BOP reagent $(1.5 \mathrm{mmol})$, TEA $(5 \mathrm{mmol})$ and the reaction mass was stirred at RT for $12 \mathrm{~h}$. Completion of the reaction was monitored by LC/MS. The reaction mass was diluted with ice cold water, white solid precipitates out was filtered and suction dried.

\section{General Procedure for the Preparation of Quinoline- 6-carboxamides, 3a-d}

To a mixture of quinoline-6-carboxylic acid, $\mathbf{3}$ ( $1 \mathrm{mmol})$ in DMF was added amine (1.5 mmol), BOP reagent (1.5 mmol), TEA ( $5 \mathrm{mmol})$ and the reaction mass was stirred at $\mathrm{RT}$ for $12 \mathrm{~h}$. Completion of the reaction was monitored by $\mathrm{LC} / \mathrm{MS}$. The reaction mass was diluted with ice cold water, white solid precipitates out was filtered and suction dried.

\section{Spectral Data}

2-Chloro- $N$-(6-methoxy-1,3-benzothiazol-2-yl)quinoline4-carboxamide, 2a

Pale Yellow Solid, mp $310-312{ }^{\circ} \mathrm{C}$; ${ }^{1} \mathrm{H}-\mathrm{NMR}$ (400 MHz, DMSO- $\left.d_{6}\right): \delta 3.83(\mathrm{~d}, \mathrm{~J}=2.40 \mathrm{~Hz}, 3 \mathrm{H}), 6.83(\mathrm{~s}$, 1H), 7.63-7.64 (m, 1H), 7.69-7.71 (m, 2H), 7.76 (t, J = $7.20 \mathrm{~Hz}, 1 \mathrm{H}), 7.93(\mathrm{~d}, \mathrm{~J}=9.60 \mathrm{~Hz}, 2 \mathrm{H}), 8.00(\mathrm{~s}, 1 \mathrm{H}), 8.07$ $(\mathrm{d}, \mathrm{J}=8.00 \mathrm{~Hz}, 1 \mathrm{H}), 12.05$ (s, 1H); Mol. Wt 369.8; LCMS -370 .

2-Chloro- $\mathrm{N}$-(4-phenyltetrahydrofuran-3-yl)quinoline4-carboxamide, $2 \mathrm{~b}$

Pale Yellow Solid, mp 223-225 ${ }^{\circ} \mathrm{C}$; ${ }^{1} \mathrm{H}-\mathrm{NMR}(400 \mathrm{MHz}$, DMSO- $\left.d_{6}\right): \delta 3.46(\mathrm{t}, \mathrm{J}=3.20 \mathrm{~Hz}, 2 \mathrm{H}), 3.65(\mathrm{t}, \mathrm{J}=3.20$ $\mathrm{Hz}, 2 \mathrm{H}), 7.35-7.42(\mathrm{~m}, 3 \mathrm{H}), 7.68(\mathrm{~s}, 1 \mathrm{H}), 7.71-7.78(\mathrm{~m}$, $2 \mathrm{H}), 7.80(\mathrm{t}, \mathrm{J}=1.20 \mathrm{~Hz}, 1 \mathrm{H}), 8.03(\mathrm{~d}, \mathrm{~J}=8.00 \mathrm{~Hz}, 1 \mathrm{H})$, $8.54(\mathrm{~d}, \mathrm{~J}=7.60 \mathrm{~Hz}, 1 \mathrm{H}), 8.79(\mathrm{~d}, \mathrm{~J}=4.80 \mathrm{~Hz}, 1 \mathrm{H}), 9.56(\mathrm{~s}$, 1H). Mol. Wt. 352.82; LCMS-353.

2-Chloro- $N$-(4-methylphenyl)quinoline-4-carboxamide, 2c

White Solid, mp $157-160{ }^{\circ} \mathrm{C}$; ${ }^{1} \mathrm{H}-\mathrm{NMR}$ (400 MHz, DMSO- $\left.d_{6}\right): \delta 2.30(\mathrm{~s}, 3 \mathrm{H}), 7.20(\mathrm{~d}, \mathrm{~J}=8.40 \mathrm{~Hz}, 2 \mathrm{H}), 7.65$ $(\mathrm{d}, \mathrm{J}=8.40 \mathrm{~Hz}, 2 \mathrm{H}), 7.71(\mathrm{q}, \mathrm{J}=8.80 \mathrm{~Hz}, 2 \mathrm{H}), 7.90(\mathrm{t}, \mathrm{J}=$ $7.20 \mathrm{~Hz}, 1 \mathrm{H}), 8.05(\mathrm{~d}, \mathrm{~J}=8.00 \mathrm{~Hz}, 1 \mathrm{H}), 8.13(\mathrm{~d}, \mathrm{~J}=8.00$ Hz, 1H), 10.73 (s, 1H); Mol wt. 296.75; LCMS-297.

2-Chloro- $N$-(3,4-dimethoxyphenyl)quinoline-4-carboxamide, $2 \mathrm{~d}$

Pale Yellow Solid, mp $165-167{ }^{\circ} \mathrm{C}$; ${ }^{1} \mathrm{H}-\mathrm{NMR}(400 \mathrm{MHz}$, DMSO- $\left.d_{6}\right): \delta 3.76(\mathrm{~d}, \mathrm{~J}=2.80 \mathrm{~Hz}, 6 \mathrm{H}), 6.97(\mathrm{~d}, \mathrm{~J}=8.80$ 
$\mathrm{Hz}, 1 \mathrm{H}), 7.30(\mathrm{dd}, \mathrm{J}=2.40,8.80 \mathrm{~Hz}, 1 \mathrm{H}), 7.46(\mathrm{~d}, \mathrm{~J}=2.40$ $\mathrm{Hz}, 1 \mathrm{H}), 7.73(\mathrm{t}, \mathrm{J}=7.20 \mathrm{~Hz}, 2 \mathrm{H}), 7.87-7.93(\mathrm{~m}, 1 \mathrm{H}), 8.04$ $(\mathrm{d}, \mathrm{J}=8.00 \mathrm{~Hz}, 1 \mathrm{H}), 8.16(\mathrm{~d}, \mathrm{~J}=8.00 \mathrm{~Hz}, 1 \mathrm{H}), 10.67(\mathrm{~s}$, 1H) mol wt. 342.77; LCMS-343.

2-Chloro- $\mathrm{N}$-(6-cyanonaphthalen-2-yl)quinoline-4-carboxamide, 2e

Pale Yellow Solid, mp $145-146{ }^{\circ} \mathrm{C}$; IR (KBr), $v, \mathrm{~cm}^{-1}$ : $3300(\mathrm{NH}), 3400(\mathrm{OH}), 2209(\mathrm{CN}), 1640(\mathrm{CO}) ;{ }^{1} \mathrm{H}-\mathrm{NMR}$ $\left(400 \mathrm{MHz}, \mathrm{DMSO}-d_{6}\right): \delta 6.67(\mathrm{~d}, \mathrm{~J}=8.00 \mathrm{~Hz}, 1 \mathrm{H}), 6.92$ $(\mathrm{d}, \mathrm{J}=4.00 \mathrm{~Hz}, 2 \mathrm{H}), 7.48(\mathrm{~s}, 1 \mathrm{H}), 7.63-7.67(\mathrm{~m}, 1 \mathrm{H}), 7.73$ $(\mathrm{d}, \mathrm{J}=8.00 \mathrm{~Hz}, 1 \mathrm{H}), 7.89(\mathrm{~d}, \mathrm{~J}=7.60 \mathrm{~Hz}, 1 \mathrm{H}), 8.23(\mathrm{~d}, \mathrm{~J}=$ $8.40 \mathrm{~Hz}, 1 \mathrm{H}), 12.05$ (s, 1H); mol wt. 357.79; LCMS-358.

2-Chloro- $N$-[1-(pyrimidin-2-yl)cyclopropyl]quinoline4-carboxamide, $2 \mathrm{f}$

Yellow Solid, mp $156-158{ }^{\circ} \mathrm{C}$; ${ }^{1} \mathrm{H}-\mathrm{NMR}(400 \mathrm{MHz}$, DMSO- $\left.d_{6}\right): \delta 1.46(\mathrm{t}, \mathrm{J}=3.20 \mathrm{~Hz}, 2 \mathrm{H}), 1.65(\mathrm{t}, \mathrm{J}=3.20$ $\mathrm{Hz}, 2 \mathrm{H}), 7.35(\mathrm{t}, \mathrm{J}=4.80 \mathrm{~Hz}, 1 \mathrm{H}), 7.68(\mathrm{~s}, 1 \mathrm{H}), 7.78(\mathrm{t}, \mathrm{J}=$ $1.20 \mathrm{~Hz}, 1 \mathrm{H}), 7.80(\mathrm{t}, \mathrm{J}=1.20 \mathrm{~Hz}, 1 \mathrm{H}), 8.03(\mathrm{~d}, \mathrm{~J}=8.00$ $\mathrm{Hz}, 1 \mathrm{H}), 8.54(\mathrm{~d}, \mathrm{~J}=7.60 \mathrm{~Hz}, 1 \mathrm{H}), 8.79(\mathrm{~d}, \mathrm{~J}=4.80 \mathrm{~Hz}$, 1H), 9.56 (s, 1H); mol wt. 324.76; LCMS-325.

N-(6-Cyanonaphthalen-2-yl)quinoline-6-carboxamide, 3a

Pale Yellow Solid, mp $138-140{ }^{\circ} \mathrm{C}$; IR (KBr), $v, \mathrm{~cm}^{-1}$ : $3310(\mathrm{NH}), 3400(\mathrm{OH}), 2200(\mathrm{CN}), 1640(\mathrm{CO}) ;{ }^{1} \mathrm{H}-\mathrm{NMR}$ $\left(400 \mathrm{MHz}, \mathrm{DMSO}-d_{6}\right): \delta 6.67(\mathrm{~d}, \mathrm{~J}=8.00 \mathrm{~Hz}, 1 \mathrm{H}), 6.92$ (d, J = 4.00 Hz, 2H), 7.48-7.40 (m, 2H), 7.63-7.67 (m, 1H), $7.73(\mathrm{~d}, \mathrm{~J}=8.00 \mathrm{~Hz}, 1 \mathrm{H}), 7.89(\mathrm{~d}, \mathrm{~J}=7.60 \mathrm{~Hz}, 1 \mathrm{H})$, $8.23(\mathrm{~d}, \mathrm{~J}=8.40 \mathrm{~Hz}, 1 \mathrm{H}), 12.05$ (s, 1H); mol wt. 323.34; LCMS-324.

N-(6-Methoxy-1,3-benzothiazol-2-yl)quinoline-6-carboxamide, $3 \mathrm{~b}$

Off-white Solid, mp $184-187^{\circ} \mathrm{C}$; IR (KBr), $v, \mathrm{~cm}^{-1}: 3200$ $(\mathrm{NH}), 1650(\mathrm{CO}), 1600$ (aromatic $\mathrm{C}=\mathrm{C}) ;{ }^{1} \mathrm{H}-\mathrm{NMR}(400$ MHz, DMSO- $\left.d_{6}\right): \delta 3.72(\mathrm{~s}, 3 \mathrm{H}), 7.07$ (dd, $\mathrm{J}=2.80,9.00$ $\mathrm{Hz}, 1 \mathrm{H}), 7.62-7.67$ (m, 3H), $8.15(\mathrm{~d}, \mathrm{~J}=8.80 \mathrm{~Hz}, 1 \mathrm{H})$, $8.37(\mathrm{~d}, \mathrm{~J}=1.60 \mathrm{~Hz}, 1 \mathrm{H}), 8.53(\mathrm{~d}, \mathrm{~J}=8.00 \mathrm{~Hz}, 1 \mathrm{H}), 8.86$ $(\mathrm{d}, \mathrm{J}=1.60 \mathrm{~Hz}, 1 \mathrm{H}), 9.90(\mathrm{q}, \mathrm{J}=0.16 \mathrm{~Hz}, 1 \mathrm{H}), 12.95$ (s, 1H); mol wt 335.37; LCMS-336.

N-(4-Methylphenyl)quinoline-6-carboxamide, 3c Pale Yellow Solid, mp $165-167^{\circ} \mathrm{C}$; IR (KBr), $v, \mathrm{~cm}^{-1}$ : $3300(\mathrm{NH}), 1650(\mathrm{CO}), 1600$ (aromatic $\mathrm{C}=\mathrm{C}$ ); ${ }^{1} \mathrm{H}-\mathrm{NMR}$ (400 MHz, DMSO- $\left.d_{6}\right): \delta 2.29$ (s, $\left.3 \mathrm{H}\right), 7.18(\mathrm{~d}, \mathrm{~J}=8.40$ $\mathrm{Hz}, 2 \mathrm{H}), 7.63$ (q, J = $4.40 \mathrm{~Hz}, 1 \mathrm{H}), 7.69$ (d, J $=8.40 \mathrm{~Hz}$, $2 \mathrm{H}), 8.23(\mathrm{~d}, \mathrm{~J}=2.00 \mathrm{~Hz}, 1 \mathrm{H}), 8.25(\mathrm{~d}, \mathrm{~J}=2.00 \mathrm{~Hz}, 1 \mathrm{H})$, $8.52(\mathrm{dd}, \mathrm{J}=1.20,8.60 \mathrm{~Hz}, 1 \mathrm{H}), 8.61(\mathrm{~d}, \mathrm{~J}=2.00 \mathrm{~Hz}, 1 \mathrm{H})$, 9.00 (q, J = $1.60 \mathrm{~Hz}, 1 \mathrm{H}), 10.40$ (s, 1H); mol wt 262.3; LCMS-263.

$\mathrm{N}$-(3,4-Dimethoxyphenyl)quinoline-6-carboxamide, 3d Pale Yellow Solid, mp 147-149 ${ }^{\circ} \mathrm{C}$; IR (KBr), $v, \mathrm{~cm}^{-1}$ :
$3300(\mathrm{NH}), 1653(\mathrm{CO}), 1600$ (aromatic $\mathrm{C}=\mathrm{C}$ ); ${ }^{1} \mathrm{H}-\mathrm{NMR}$ $\left(400 \mathrm{MHz}, \mathrm{DMSO}-d_{6}\right): \delta 3.75(\mathrm{~s}, 3 \mathrm{H}), 3.77(\mathrm{~s}, 3 \mathrm{H}), 6.95$ $(\mathrm{d}, \mathrm{J}=8.80 \mathrm{~Hz}, 1 \mathrm{H}), 7.50(\mathrm{~d}, \mathrm{~J}=2.40 \mathrm{~Hz}, 1 \mathrm{H}), 7.62-7.65$ $(\mathrm{m}, 1 \mathrm{H}), 8.12(\mathrm{~d}, \mathrm{~J}=8.80 \mathrm{~Hz}, 1 \mathrm{H}), 8.25(\mathrm{dd}, \mathrm{J}=2.00,8.80$ $\mathrm{Hz}, 1 \mathrm{H}), 8.51(\mathrm{~d}, \mathrm{~J}=5.60 \mathrm{~Hz}, 1 \mathrm{H}), 8.61(\mathrm{~d}, \mathrm{~J}=2.00 \mathrm{~Hz}$, $1 \mathrm{H}), 8.99-9.11(\mathrm{~m}, 1 \mathrm{H}), 10.34$ (s, $1 \mathrm{H})$; mol wt 308.33; LCMS-309.

\section{Determination of Minimum Inhibitory Concentration (MIC)}

The minimum inhibitory concentrations of the synthesized compounds were determined by agar dilution method. ${ }^{13}$ The growth media, nutrient agar was first prepared in a usual method and sterilized by autoclaving. The sterilized media was allowed to cool to $50{ }^{\circ} \mathrm{C}$ and $9 \mathrm{ml}$ of the molten agar was added to test tubes which contained $1 \mathrm{ml}$ of different concentration of the test compounds and the control (DMSO). The mixture of the media and the test drugs were thoroughly mixed and poured into pre-labeled sterile petri dishes on a level surface. Additional petri dishes containing only the growth media were prepared in the same way so as to serve for comparison of growth of the respective organisms. The concentrations of the compounds used for test were ranging from 0.5 to $2.5 \mathrm{mg} / \mathrm{ml}$. The plates were dried at room temperature in laminar air flow for 30 min. The suspensions of the respective microorganisms were inoculated into the series of agar plates using standard loop. The plates were then incubated at $37^{\circ} \mathrm{C}$ for $24 \mathrm{~h}$. The lowest concentration which inhibited the growth of the respective organisms was taken as MIC. All tests were carried out in duplicate and results are shown in Table 3.

Acknowledgments. The authors G R Vijayakumar and D B Aruna Kumar are thankful to Registrar and ViceChancellor of Tumkur University, Tumkur, India for providing laboratory facility. And the publication cost of this paper was supported by the Korean Chemical Society.

\section{REFERENCES}

1. Roma, G.; Grossi, G.; Di Braccio, M.; Piras, D.; Ballabeni, V.; Tognolini, M.; Bertoni, S.; Barocelli. E. Eur. J. Med. Chem. 2008, 43, 1665.

2. Venkat Reddy, G.; et al. Eur. J. Med. Chem. 2009, 44, 1570.

3. Tomassoli, I.; et al. Eur. J. Med. Chem. 2011, 46, 1.

4. Sliman, F.; Blairvacq, M.; Durieu, E.; Meijer, L.; Rodrigo, J.; Desmaële, D. Bioorg. Med. Chem. Lett. 2010, 20, 2801.

5. Zhang, J.; Ke, X.; Tu, C.; Lin, J.; Ding, J.; Lin, L.; Fun, H. K.; You, X.; Guo, Z. Biometals. 2003, 16, 485. 
6. Tsuji, K.; Spears, G. W.; Nakamura, K.; Tojo, T.; Seki, N.; Sugiyama, A.; Matsuo, M. Bioorg. Med. Chem. Lett. 2002, $12,85$.

7. Bu, X.; Chen, J.; Deady, L. W.; Smith, C. L.; Baguley, B. C.; Greenhalgh, D.; Yang, S.; Denny, W. A. Bioorg. Med. Chem. Lett. 2005, 13, 3657.

8. Liu, J.-Y.; Yu, H.-L.; Quan, Z.-S.; Cui, X.; Piao, H.-R. Bioorg. Med. Chem. Lett. 2009, 19, 2392.

9. Belloli, S.; et al. Neurochem. International, 2004, 44,
433.

10. Wentland, M. P.; et al. Drug Des Discov. 1997, 15, 25.

11. Pelczar, M. J.; Chan, E. C. S. Laboratory Exercise in Microbiology; McGraw-Hill Inc: USA, 1997, Vo. 17, p 301.

12. Cruickshank, R.; Duguid, J. P.; Marmion, B. P.; Swain, R. H. A. Med. Microbiol. 1989, 201.

13. EUCAST Definitive Document. Determination of Minimum Inhibitory Concentrations (MICs) of Antimicrobial Agents by Agar Dilution. 2000, Vol. 6, pp 509-515. 